\title{
ANÁLISE GEOESPACIAL DA LEISHMANIOSE TEGUMENTAR AMERICANA NO ESTADO DE SÃO PAULO: UMA CONTRIBUIÇÃO À GEOGRAFIA DA SAÚDE
}

\author{
Danilo Carneiro Valente ${ }^{1}$ \\ Marcos César Ferreira ${ }^{2}$
}

Resumo: A Leishmaniose Tegumentar Americana (LTA) é uma doença infectoparasitária causada pela infecção por protozoários do gênero Leishmania. A maioria dos casos ocorrem em áreas próximas à fragmentos de matas e em bairros rurais. Os objetivos deste artigo são: mapear as áreas de alto risco de LTA e analisar a associação espacial entre taxa de incidência de LTA e determinantes geográficos da doença no estado de São Paulo, como percentual de população rural, percentual de cobertura vegetal nativa e renda per capita do município. Para isso, utilizamos a técnica de suavização bayesiana empírica de taxas brutas e aplicação da técnica de autocorrelação espacial bivariada local. Os resultados mostraram que o principal aglomerado de alto risco de LTA localiza-se no Vale do Ribeira. As taxas mais altas de incidência estão associadas espacialmente a municípios com alto percentual de população rural, alto percentual de cobertura vegetal primitiva e baixa renda per capita.

Palavras-chave: Análise geoespacial; Leishmaniose Tegumentar Americana, Determinantes geográficos, São Paulo.

\section{GEOSPATIAL ANALYSIS OF AMERICAN TEGUMENTARY LEISHMANIASIS IN THE STATE OF SÃO PAULO: A CONTRIBUTION TO HEALTH GEOGRAPHY}

Abstract: American tegumentary leishmaniasis (ATL) is an infectious and parasitic disease caused by protozoa of the genus Leishmania. Most cases occur in areas close to forest fragments and in rural neighborhoods. The objectives of this study were to map high-risk areas for ATL and to analyze the spatial association between the ATL incidence rate and the geographic determinants of the disease in the state of São Paulo, such as the percentage of rural population, percentage of native vegetation cover and per capita income of the municipality. For this, we used the empirical Bayesian smoothing technique of gross rates and the local bivariate spatial autocorrelation technique. The results showed that the main high-risk cluster of ATL occurs in Vale do Ribeira. The highest incidence rates are spatially associated with municipalities with a high percentage of rural population and primitive vegetation cover and a low per capita income.

\footnotetext{
${ }^{1}$ Mestrando em Geografia pela Unicamp (Campinas/SP). Email: daniloc.valente@gmail.com

${ }^{2}$ Professor Associado III do Instituto de Geociências - Unicamp (Campinas/SP). Email: macferre@ unicamp.br
} 
Keywords: Geospatial analysis, American Tegumentary Leishmaniais, Geographic determinants, São Paulo.

\section{INTRODUÇÃO}

A Leishmaniose Tegumentar Americana (LTA) é uma doença infectoparasitária que acomete anualmente entre 900 mil a 1,2 milhões de pessoas no mundo (ALI-AKBARPOUR et al., 2012; BRASIL, 2013). Considerada pela Organização Mundial de Saúde (OMS) um problema de saúde pública em 88 países, a LTA é uma entre as seis doenças infecciosas mais importantes do mundo, com ampla distribuição geográfica, e casos registrados na Arábia Saudita, Brasil, Irã, Peru e Síria (GOSCH et al., 2017). Nas Américas são registrados casos desde os Estados Unidos até a Argentina, exceto no Chile e no Uruguai (LAINSON, 1981; RODRÍGUEZ; DÍAZ; PÉRES, 2013).

A LTA é causada pela infecção por protozoários do gênero Leishmania, e é transmitida aos humanos por meio da picada de fêmeas de diversas espécies de mosquitos (vetores da doença) flebotomíneos, dos gêneros Lutzomyia e Psychodopygus (BRASIL, 2006). A doença causa lesões graves na estrutura da pele e das mucosas, principalmente nas partes mais expostas do corpo, como os membros superiores e inferiores. O habitat dos vetores da LTA e os fatores geográficos a eles associados, apresentam papel importante na evolução e transmissão desta doença (MARCHI et al., 2019).

A LTA é registrada em todas as regiões do Brasil, cujas características específicas de ocupação do território e dos ecossistemas favorecem a existência de habitats dos vetores e de ambientes onde se localizam diferentes hospedeiros do protozoário do gênero Leishmania. A doença ocorre com maior frequência em ambientes florestais antropizados, associados a desmatamento, construção de estradas e urbanização de áreas próximas a florestas. Estes fatores têm favorecido o desenvolvimento do ciclo peridomiciliar da LTA (DEDET, 1999; apud NEGRÃO; FERREIRA, 2014).

A maioria dos casos de LTA ocorrem em áreas povoadas, situadas próximas a unidades de conservação, fragmentos de matas e em bairros rurais. O mosquito vetor da LTA se reproduz com maior facilidade em áreas que apresentam umidade e temperatura elevadas, onde os fatores ambientais contribuem para desenvolvimento dos insetos. A transmissão da doença também é influenciada por fatores sociais e econômicos (WHO, 2010; MENDES et al., 2016).

O crescimento migratório aliado ao processo histórico de organização espacial nas últimas décadas, têm propiciado a proliferação de áreas com alto risco de infecção por Leishmania também em áreas urbanas brasileiras, associadas a áreas verdes e/ou como consequência do desequilíbrio ecológico, evidenciadas em áreas periurbanas ou associadas ao desmatamento (NEGRÃO; FERREIRA, 2014).

No estado de São Paulo a LTA evoluiu junto ao histórico de desmatamento associado a fronteiras agrícolas no centro-oeste do estado, ligado a agricultura. $\mathrm{Na}$ década de 1970, verificou-se o aumento do número de casos no estado, com destaque para a região sul. Nas últimas décadas foram encontrados novos focos de transmissão em ambientes periurbanos, com registros espalhados por diversas regiões do estado de São Paulo (SHIMABUKURO et al., 2010; MORETTI; FERREIRA, 2011).

Em estudo realizado no estado do Amazonas, Guerra et al. (2015) constataram que $43,7 \%$ dos pacientes acometidos pela LTA tinham ocupação profissional relacionadas à agricultura, silvicultura, caça e pesca, e apenas $0,1 \%$ eram técnicos de 
nível médio. Ainda segundo os autores, a descoberta de novas áreas de mineração, o início da exploração de petróleo e a construção de oleodutos, contribuíram para o desmatamento e para o contato de trabalhadores com os vetores da LTA. Resende (2004) realizou um estudo eco-epidemiológico da LTA na microrregião de CaratingaMG, utilizando dados da série temporal de 1966 a 2003, e concluiu que $78 \%$ dos casos da doença ocorreram em ambientes rurais.

Bezerra et al. (2018) desenvolveram um extenso estudo sobre a LTA e a leishmaniose visceral nas 27 unidades da federação. Constataram que a taxa de incidência ajustada à idade cresceu entre 52,9\% e 108\%, no período de 1990 a 2016. Segundo os autores, o crescimento dos casos pode estar relacionado à dificuldade da adoção de medidas de prevenção e tratamento, e ao fato desta doença ainda ser negligenciada pelos órgãos de saúde pública no país.

Fatores ambientais relacionados à LTA na Colômbia foram investigados por Valderrama-Ardila et al. (2010). Os pesquisadores concluíram que a temperatura e o percentual de cobertura vegetal estavam associados à taxa de incidência da LTA. Mollalo et al. (2015) mapearam aglomerados de LTA no Irã, utilizando técnicas de autocorrelação espacial e o índice de Moran. Os autores constataram que os aglomerados mais significativos estavam localizados predominantemente no norte do país, em áreas de baixas altitudes, em climas áridos e semiáridos, e onde residem populações em condições socioeconômicas de pobreza.

Entendemos que a análise espacial da LTA deve ser pautada por abordagens geográficas integradas, baseadas no paradigma da epidemiologia da paisagem. $\mathrm{O}$ conceito de epidemiologia da paisagem, proposto por Pavloskiy (1948; 1966), descreve como a dinâmica temporal do hospedeiro, do vetor e dos patógenos interagem espacialmente dentro de um ambiente que é permissivo à transmissão de uma determinada doença (REISEN, 2010). Ainda segundo Reisen, o foco de transmissão (ou nidus) é caracterizado pela vegetação, clima, latitude, altitude e geologia, geomorfologia, entre outros. Os nidus da LTA são formados por uma complexa associação espacial entre fatores ambientais, socioeconômicos e demográficos.

As mudanças históricas na estrutura da paisagem no estado de São Paulo ao longo do século XX podem ter afetado a epidemiologia espacial da LTA. Novas formas de uso agrícola das terras, ou de habitações e bairros situados próximos a fragmentos florestais e unidades de conservação, podem ter contribuído para a dispersão dos nidus da LTA, trazendo-os para áreas peridomésticas, aumentando assim, o risco de transmissão humana.

\section{OBJETIVOS}

Os objetivos deste trabalho foram: aplicar métodos e técnicas de análise geoespacial para mapear áreas endêmicas de Leishmaniose Tegumentar Americana (LTA) no estado de São Paulo; identificar as áreas de alto risco de LTA no estado; analisar a associação espacial entre a taxa de incidência de LTA e determinantes geográficos da doença, e caracterizar as unidades de paisagem com alto risco de LTA no estado de São Paulo. 


\title{
MATERIAIS E METODOLOGIA
}

\author{
MATERIAIS
}

\section{DADOS EPIDEMIOLÓGICOS}

Os dados relativos ao número de casos confirmados de Leishmaniose Tegumentar Americana (LTA) por município do estado de São Paulo, correspondente a uma série temporal de 12 anos (2007 a 2018), foram obtidos junto ao Sistema de Informação de Agravos de Notificações (SINAN, 2020), do Ministério da Saúde do Brasil.

\section{DADOS DEMOGRÁFICOS E SOCIOECONÔMICOS}

Os dados anuais sobre a população total no período de 2007 a 2018, e o total da população rural em 2020 (PRU), por município do estado, foram obtidos no sítio do Sistema Estadual de Análise de Dados (SEADE, 2020). Nesta mesma fonte foram coletados também, os dados sobre a renda per capita (RPC) municipal para o ano de 2010.

\section{DADOS SOBRE A COBERTURA VEGETAL NATIVA}

Foram utilizados dados do Percentual de Cobertura Vegetal nativa (PCV) por município do estado de São Paulo, obtidos no Inventário Florestal do Estado de São Paulo de 2020 (SÃO PAULO, 2020). Este levantamento foi realizado pelo Instituto Florestal, com base em mapeamento realizado a partir de imagens orbitais de alta resolução espacial, referentes ao período de 2017 a 2019.

\section{DADOS CARTOGRÁFICOS VETORIAIS}

Foi utilizada a base cartográfica dos municípios do estado de São Paulo, produzida pelo Instituto Brasileiro de Geografia e Estatística (IBGE, 2020). Esta base, em formato vetorial, em arquivo shapefile poligonal, contém os limites territoriais dos municípios paulistas.

\section{MAPAS FÍSICO GEOGRÁFICOS}

Foi utilizado o seguinte conjunto de mapas temáticos para a caracterização fisicogeográfica dos aglomerados de alto risco de leishmaniose tegumentar americana em São Paulo:

a) Geomorfologia: Divisão geomorfológica do estado de São Paulo (IPT, 1981) e Mapa geomorfológico do estado de São Paulo (ROSS; MOROZ, 1996)

b) Fitofisionomias: Inventário florestal do estado de São Paulo, Instituto Florestal (SÃO PAULO, 2020)

c) Pluviosidade: Clima do estado de São Paulo (MARTINELLI, 2010)

d) Temperatura: Isotermas anuais do estado de São Paulo (CIIAGRO, 1974).

e) Umidade relativa do ar: Umidade relativa anual do estado de São Paulo (CIIAGRO, 1974). 


\section{METODOLOGIA}

MAPAS COROPLÉTICOS DOS DETERMINANTES RENDA PER CAPITA (RPC), PERCENTUAL DE POPULAÇÃO RURAL (PRU) E PERCENTUAL DE COBERTURA VEGETAL NATIVA POR MUNICÍPIO (PCV)

Esta e as demais etapas de análise espacial da pesquisa foram realizadas no software de análise exploratória de dados espaciais GeoDa v.1.14.07 (ANSELIN, 2019). Primeiramente, a distribuição espacial das variáveis RPC, PRU e PCV foi representada por meio de mapas coropléticos, utilizando-se a técnica de classificação de dados baseada em quantis, em cinco classes. Esta técnica permitiu distribuir espacialmente proporções similares de municípios por classe do mapa, em ordem crescente da variável. A partir destes mapas, foi possível identificar a localização, no estado, dos quantis superiores e inferiores de cada variável espacial analisada.

\section{MAPEAMENTO DAS ÁREAS DE RISCO DE LEISHMANIOSE TEGUMENTAR AMERICANA NO ESTADO DE SÃO PAULO}

Para o mapeamento das áreas de risco LTA no estado foi utilizada a técnica de suavização de taxas brutas denominada Suavização Bayesiana Empírica (EBS) (ANSELIN et al., 2010). Esta técnica possibilitou a remoção da instabilidade da variância dos dados populacionais presente em municípios que apresentaram população total pequena, por meio de suavização das taxas de incidência previamente calculadas. Com base nesta técnica, os municípios que possuem populações pequenas, expostas ao risco de LTA, tiveram suas taxas de incidência brutas ajustadas, enquanto, nos municípios com áreas e populações maiores, a taxa de incidência bruta de LTA foi pouco alterada.

Através da técnica EBS calculamos a média ponderada entre a taxa de incidência bruta de LTA em cada município, e a taxa de incidência bruta média de LTA no estado de São Paulo. Para isto, utilizamos o filtro espacial de vizinhança Queen de $1^{\underline{a}}$ ordem (ANSELIN et al., 2010). Este filtro que define como municípios vizinhos a um determinado município $M$, os municípios cujos limites territoriais fazem contato direto com $M$, seja em um, ou mais lados do polígono territorial. Os valores das taxas de incidência suavizadas de LTA calculadas por município (INC_EBS) foram utilizados para a construção do mapa de risco de LTA. Neste mapa os municípios representados em tonalidades de azul (abaixo de $25 \%$, e entre $25 \%$ e $50 \%$ ) foram os que apresentaram baixo risco de LTA em relação à média estadual; os municípios representados em tonalidades de marrom apresentaram alto risco em relação a média estadual ( $50 \%$ a $75 \%$; e acima de $75 \%)$.

ANÁLISE DE AUTOCORRELAÇÃO ESPACIAL BIVARIADA LOCAL ENTRE A TAXA DE INCIDÊNCIA SUAVIZADA DE LTA E OS DETERMINANTES RENDA PER CAPITA (RPC), PERCENTUAL DE POPULAÇÃO RURAL (PRU) E PERCENTUAL DE COBERTURA VEGETAL NATIVA (PCV) POR MUNICÍPIO

Utilizamos a técnica de análise de autocorrelação espacial bivariada local (ANSELIN et al., 2010) para identificar a associação espacial entre a taxa de incidência suavizada de LTA (INC_EBS) no município, e os valores médios dos determinantes RPC, PRU e PCV, calculados nos municípios vizinhos. A vizinhança municipal foi definida com base na técnica Queen de pesos de contiguidade espacial 
entre polígonos, selecionando-se a opção de contiguidade de primeira ordem (ANSELIN et al., 2010).

O valor de INC_EBS no município $M$ foi relacionado aos valores dos determinantes RPC, PRU e PCV, medidos em municípios vizinhos e contíguos, situados em contato direto com $M$. Por meio da análise de autocorrelação espacial bivariada local foram produzidos mapas dos aglomerados formados por distritos com altos valores para a variável INC_EBS, que estavam circundados por distritos com altos valores para cada um dos determinantes RPC, PRU e PCV. Estes aglomerados foram representados na classe $\mathrm{H}-\mathrm{H}$ (ou High-High).

Foi calculado o valor do índice de autocorrelação bivariada local I de Moran para cada associação espacial entre INC_EBS e os determinantes analisados, também foram mapeados os demais aglomerados do tipo L-L (Low-Low), L-H (LowHigh) e H-L (High-Low), considerando-se sempre, como nível de significância estatística, $p$-value $<0.05$.

\section{RESULTADOS}

\section{RISCO DE OCORRÊNCIA DE CASOS LEISHMANIA TEGUMENTAR AMERICANA}

No período de 2007 a 2018 foram notificados 4.388 casos de LTA no estado de São Paulo, com as maiores frequências de casos observadas em 2015 (478 casos, ou $10,89 \%$ ) e 2009 (446 casos ou 10,16\%) (Figura 1).

Figura 1. Números totais de casos anuais de leishmaniose tegumentar americana em municípios do estado de São Paulo, entre 2007 e 2018.

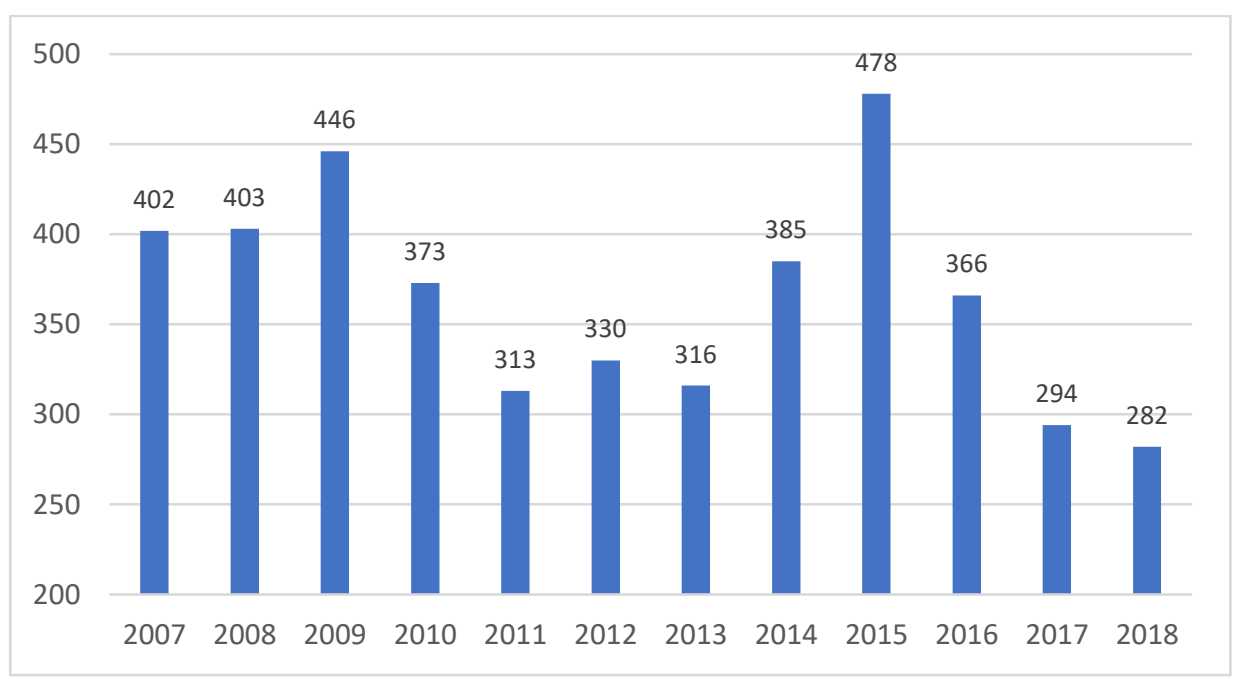

Fonte: adaptado de SINAN (2020).

O diagrama da Figura 2 representa os valores dos desvios anuais em relação à mediana dos casos de LTA no período 2007-2018. Nota-se que o maior desvio positivo ocorreu em $2015(0,294)$ e o maior desvio negativo foi constatado em 2018 ($0,237)$. Observa-se ainda que o período de 2007 a 2010 apresentou uma sequência contínua de desvios positivos, enquanto o período de 2011 a 2013 apresentou uma sequência contínua de desvios negativos.

A Figura 3 mostra o mapa de risco de LTA na população dos municípios do estado, calculado com base no total de casos acumulados no período e a média da população 
total nos respectivos municípios, no período 2007-2018, utilizando-se a técnica de suavização empírica bayesiana (EBS).

Figura 2. Desvios anuais em relação à mediana do número total de casos de leishmaniose tegumentar americana acumulados entre 2007 e 2018, em municípios do estado de São Paulo.

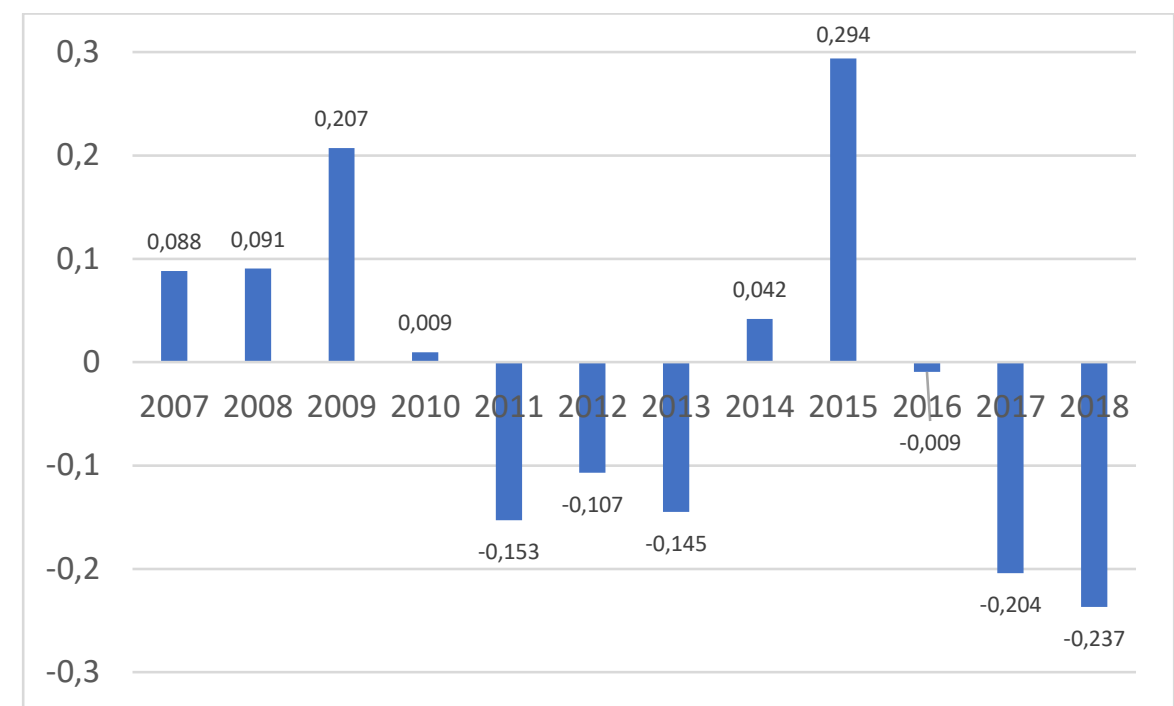

Fonte: Elaborado pelos autores (2021) a partir de dados do SINAN (2020).

Figura 3. Mapa do risco de leishmaniose tegumentar americana no estado de São Paulo, calculado pela técnica de suavização bayesiana empírica (EBS), a partir de dados do período de 2007 a 2018.

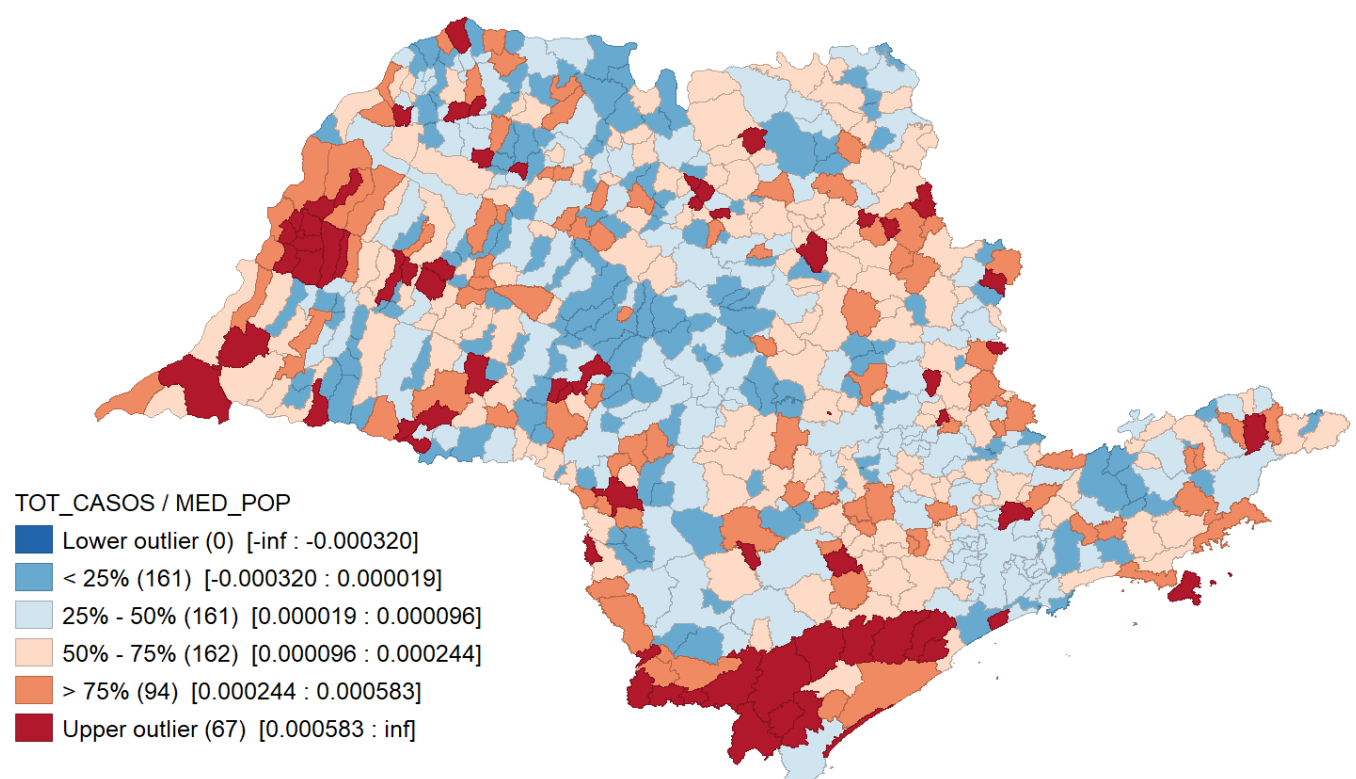

Fonte: Elaborado pelos autores (2021).

Podemos observar no mapa da Figura 3, e nos detalhes apresentados nas Figuras 4 e 5, a ocorrência de dois aglomerados de municípios com alto risco de LTA, um deles situado na região sul do estado, no Vale do Ribeira, e o outro no extremo oeste do estado. 
O aglomerado do Vale do Ribeira é formado pelos municípios de Itariri, Pedro de Toledo, Miracatu, Juquiá, Sete Barras, Eldorado, Jacupiranga, Cajati, Barra do Turvo, Iporanga, Iguape, Cananéia, Itaoca, Itapirapuã Paulista, Bom Sucesso de Itararé, Barra do Chapéu e Apiaí (Figura 4). O aglomerado de alto risco do Extremo Oeste é composto por Muritinga do Sul, Nova Independência, São João do Pau d'Alho, Tupi Paulista, Dracena, Panorama, Paulicéia, Castilho, Andradina, Guaraçaí, Pacaembu, Mirandópolis Ouro Verde, Marabá Paulista e Teodoro Sampaio (Figura 5).

Figura 4. Detalhe do mapa do risco de leishmaniose tegumentar americana do estado de São Paulo, enfatizando o aglomerado com alto risco (maior que 50\%) situado no vale do Ribeira, ao sul do estado.

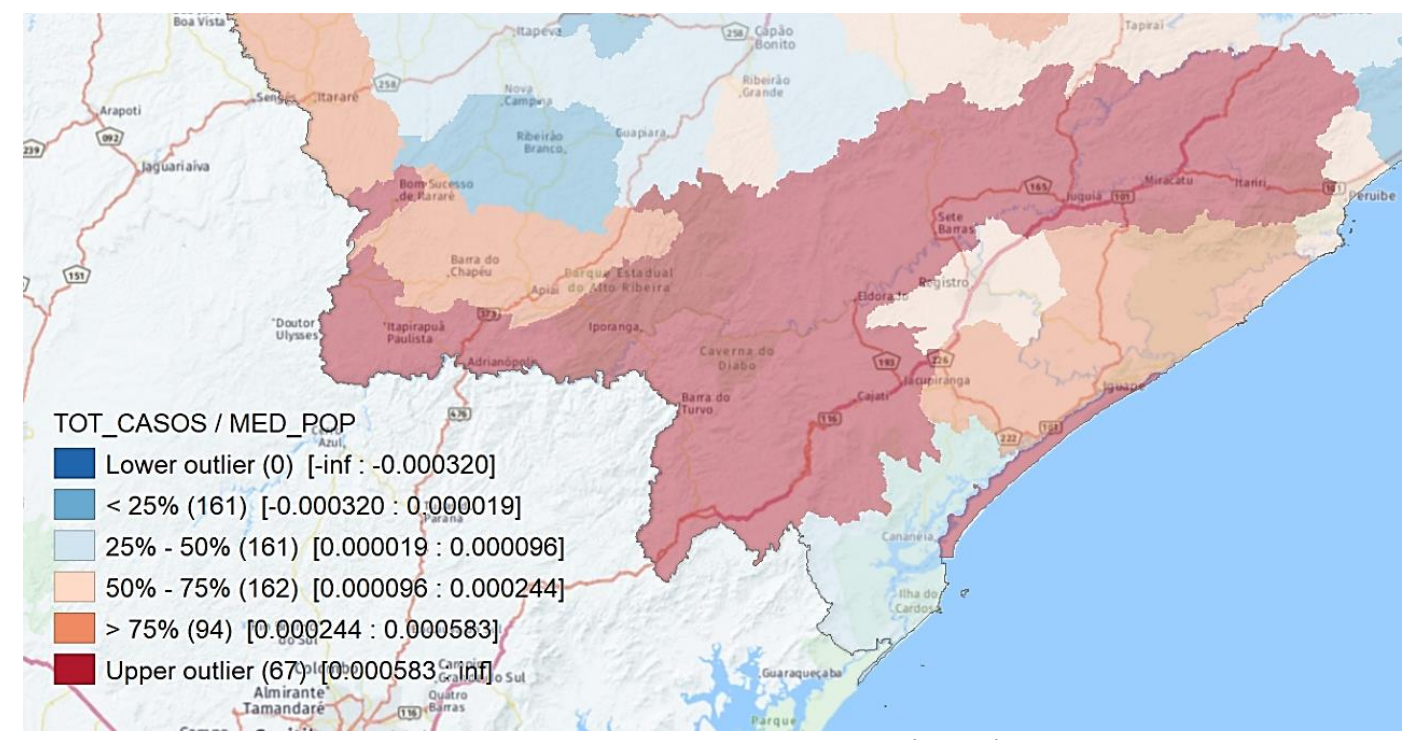

Fonte: Elaborado pelos autores (2021).

Figura 5. Detalhe do mapa do risco de leishmaniose tegumentar americana do estado de São Paulo, enfatizando o aglomerado de municípios com alto risco (maior que $50 \%$ ), localizado no extremo oeste do estado.

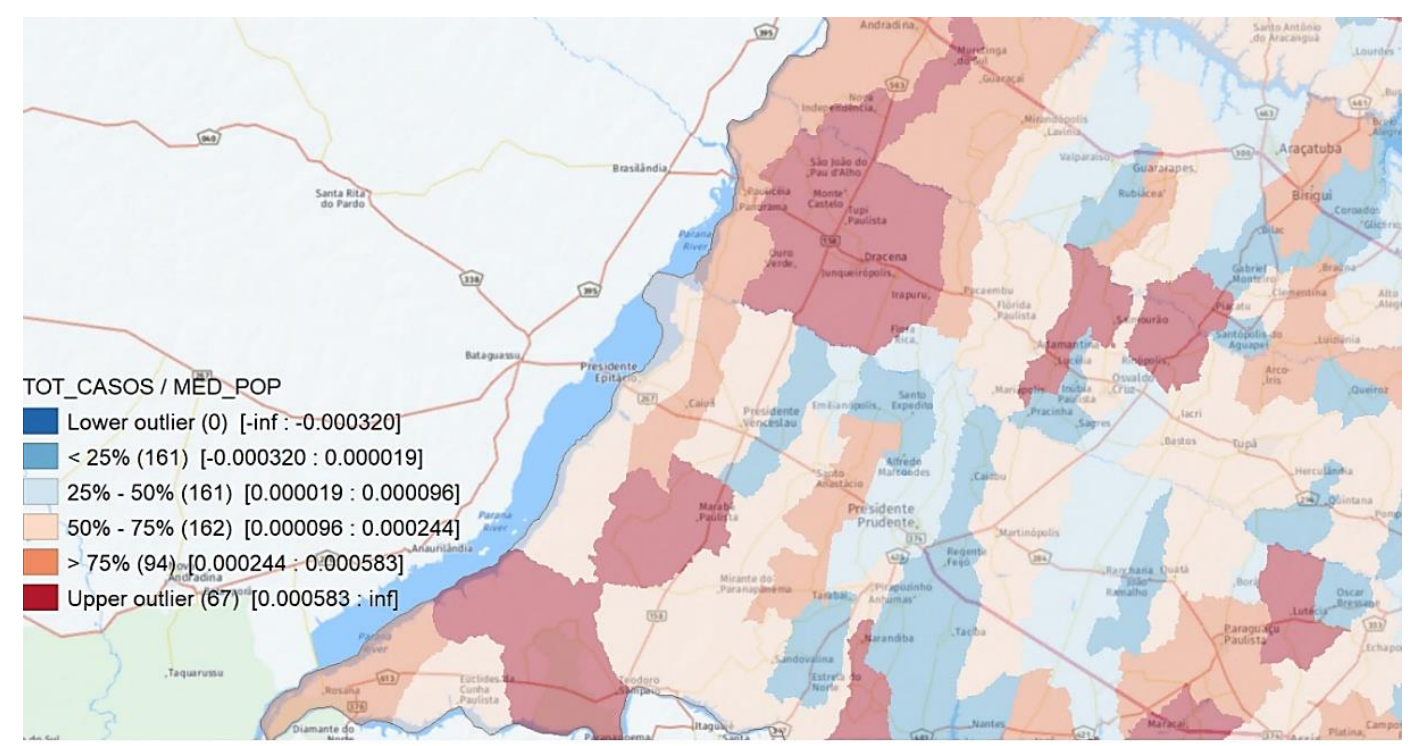

Fonte: Elaborado pelos autores (2021). 
Os mapas das variáveis percentual de cobertura vegetal primitiva em 2019 (PCV), percentual de população rural em 2020 (PRU) e renda per capita em 2010 (RPC), por município, são mostrados na Figura 6.

Figura 6. Mapas do percentual de cobertura vegetal primitiva em 2019 (A), percentual de população rural em 2020 (B) e renda per capita em 2010.
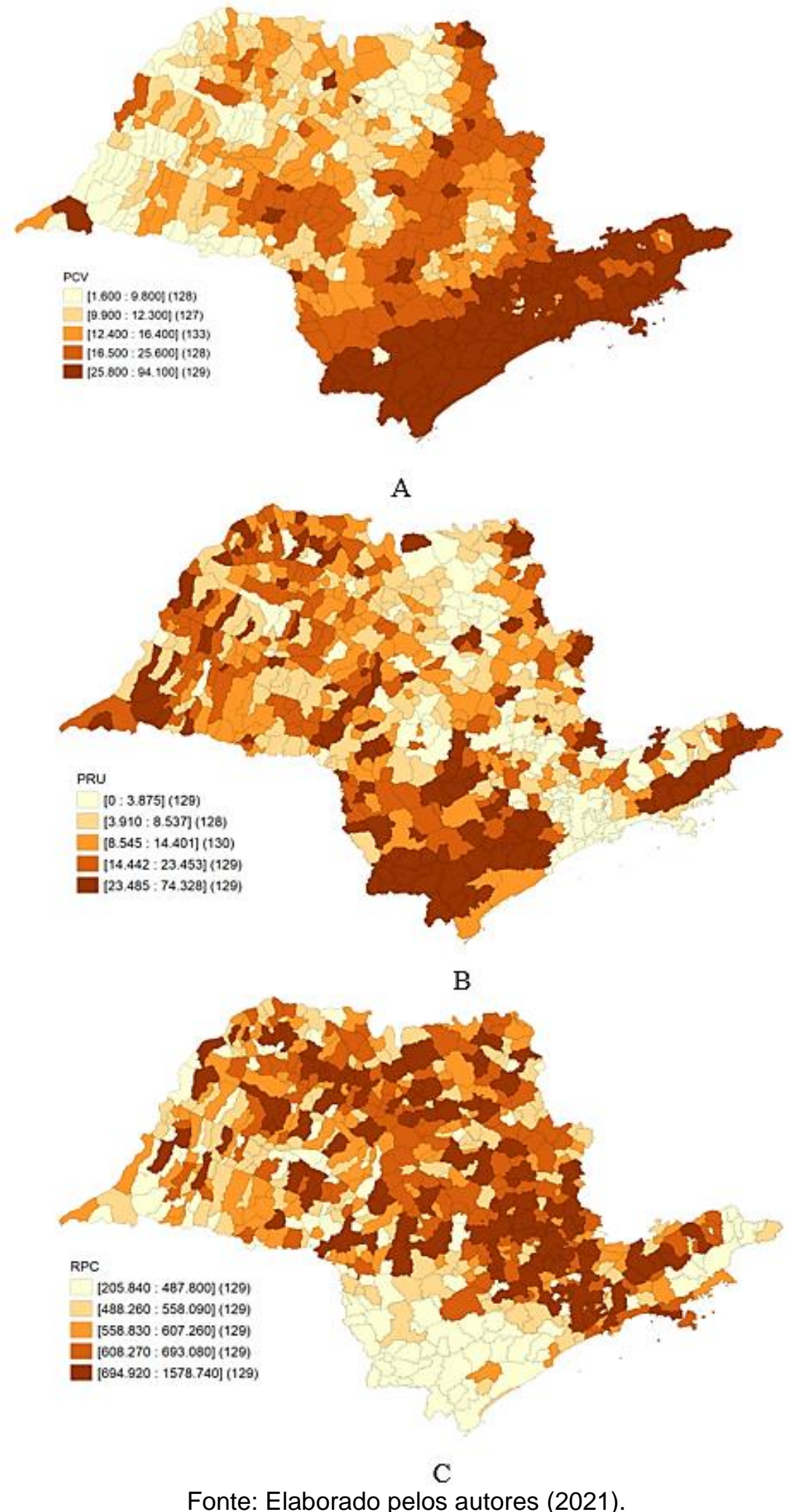

Fonte: Elaborado pelos autores (2021). 


\section{ANÁLISE DE AUTOCORRELAÇÃO ESPACIAL BIVARIADA LOCAL}

\section{RENDA PER CAPITA E LTA}

A Figura 7 mostra a distribuição espacial dos aglomerados de municípios formados pela associação espacial entre a taxa de incidência de LTA espacialmente suavizada (INC_EBS) e a renda per capita municipal (RPC). Identificamos associação espacial negativa e estatisticamente significante entre INC_EBS e RPC $(I=-0,171$; $p<0,05)$. No mapa da Figura 7 podemos observar dois aglomerados importantes de taxa de incidência de LTA associados à RPC.

$\mathrm{O}$ aglomerado $\mathrm{H}-\mathrm{L}$, formado por municípios que apresentaram alta taxa de incidência e estavam circundados por municípios com baixa renda per capita, está localizado predominantemente no Vale do Ribeira, composto por Pedro de Toledo, Miracatu, Juquiá, Sete Barras, Eldorado, Cajati, Barra do Turvo, Iporanga, Itaoca, Apiaí, Barra do Chapéu, Itapirapuã Paulista e Bom Sucesso do Itararé. Além destes municípios, este aglomerado também inclui Ubatuba, no litoral norte do estado, e Teodoro Sampaio, no Pontal do Paranapanema. Circundando o aglomerado H-L, distribuem-se municípios do aglomerado L-L, que apresentaram baixas taxas de incidência e estavam associados a municípios com baixa renda per capita. Destacase ainda, no mapa da Figura 7, o aglomerado L-H, composto por municípios com baixa taxa de incidência de LTA, contíguos a municípios com alta renda per capita.

Figura 7. Mapa dos aglomerados de associação espacial entre a taxa de incidência suavizada de leishmania tegumentar americana (INC_EBS) e a renda per capita (RPC), obtido pela técnica de análise de autocorrelação bivariada local.

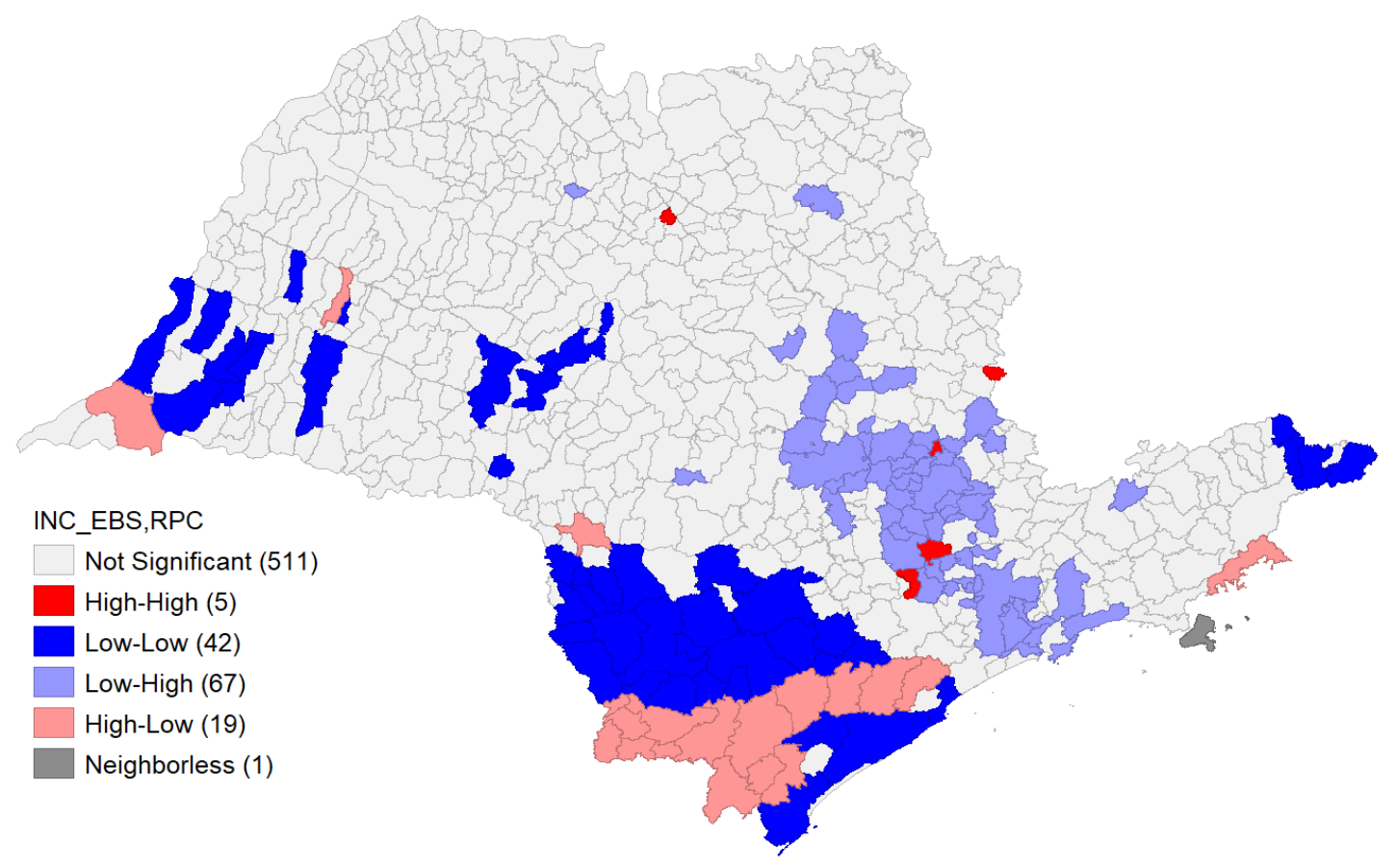

Fonte: Elaborado pelos autores (2021).

O aglomerado L-H distribui-se desde a Baixada Santista e a região metropolitana de São Paulo, passando por municípios situados no corredor das rodovias Anhanguera-Bandeirantes, na região metropolitana de Campinas, até os municípios de Analândia e Descalvado, nas Cuestas Basálticas. 


\section{PERCENTUAL DE COBERTURA VEGETAL NATIVA E LTA}

O mapa da Figura 8 representa a distribuição dos aglomerados de associação espacial entre a taxa de incidência de LTA espacialmente suavizada (INC_EBS) e o percentual de cobertura vegetal nativa no município (PCV). Constatamos uma associação espacial positiva e estatisticamente significante entre INC_EBS e PCV no estado de São Paulo $(I=0,267 ; p<0,05)$. No mapa da Figura 8 observa-se que a maioria dos municípios do sul do vale do Ribeira, que pertencem ao aglomerado $\mathrm{H}-\mathrm{H}-$ ou seja, municípios com alta taxa de incidência de LTA, circundados a municípios com alto percentual de cobertura vegetal nativa -, também fazem parte do aglomerado $\mathrm{H}$ L do mapa da Figura 7 (associação entre alta taxa de incidência de LTA e baixa renda per capita).

Figura 8. Mapa dos aglomerados de associação espacial entre a taxa de incidência suavizada de leishmania tegumentar americana e o percentual de cobertura vegetal nativa (PCV), obtido pela técnica de análise de autocorrelação bivariada local.

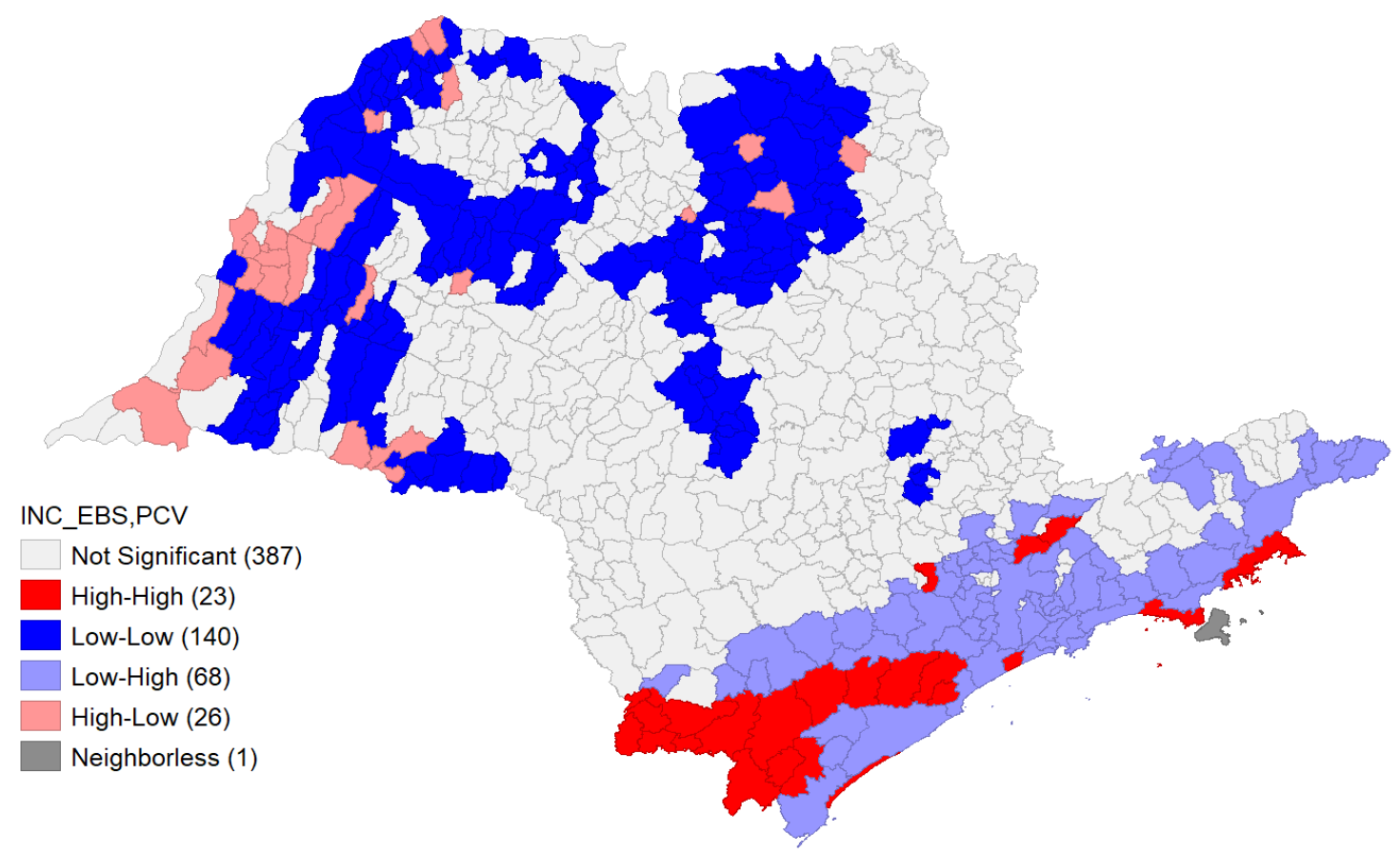

Fonte: Elaborado pelos autores (2021).

Nota-se também que o aglomerado $\mathrm{H}$-H do mapa da Figura 8 inclui municípios isolados, localizados nos litorais norte (Ubatuba e São Sebastião) e sul (Ilha Comprida e Itariri), e no Planalto Atlântico (Mairinque, Mairiporã e Nazaré Paulista). Ainda no mapa da Figura 8, podemos observar claramente um extenso aglomerado, do tipo L$\mathrm{H}$, ou seja, municípios com baixa taxa de incidência de LTA, associados espacialmente a municípios com alto percentual de cobertura vegetal nativa, que se distribui por quase todo Planalto Atlântico, desde as proximidades do Vale do Ribeira até a região leste do estado.

Outro aglomerado identificado no mapa da Figura 8 é o do tipo L-L, caracterizado pela associação espacial entre baixa taxa de incidência e baixo percentual de cobertura vegetal nativa. Com 140 municípios, distribuídos pelas regiões norte e noroeste do estado. $\mathrm{O}$ aglomerado do tipo $\mathrm{H}-\mathrm{L}$ é formado por municípios onde foi observada a associação espacial entre alta taxa de incidência de 
LTA e baixo percentual de cobertura vegetal nativa. Este aglomerado está localizado no Planalto Ocidental, se estendendo desde os municípios de Mirandópolis, Guaraçaí e Dracena, até Teodoro Sampaio, no Pontal do Paranapanema.

\section{PERCENTUAL DE POPULAÇÃO RURAL E LTA}

O mapa da Figura 9 mostra os aglomerados de associação espacial entre a taxa de incidência de LTA espacialmente suavizada (INC_EBS) e o percentual de população rural no município (PRU). Constatamos a ocorrência de associação espacial positiva e estatisticamente significante entre INC_EBS e PRU no estado de São Paulo $(I=0,211 ; p<0,05)$.

Figura 9. Mapa dos aglomerados de associação espacial entre a taxa de incidência suavizada de leishmania tegumentar americana e o percentual de população rural (PRU), obtido pela técnica de análise de autocorrelação bivariada local.

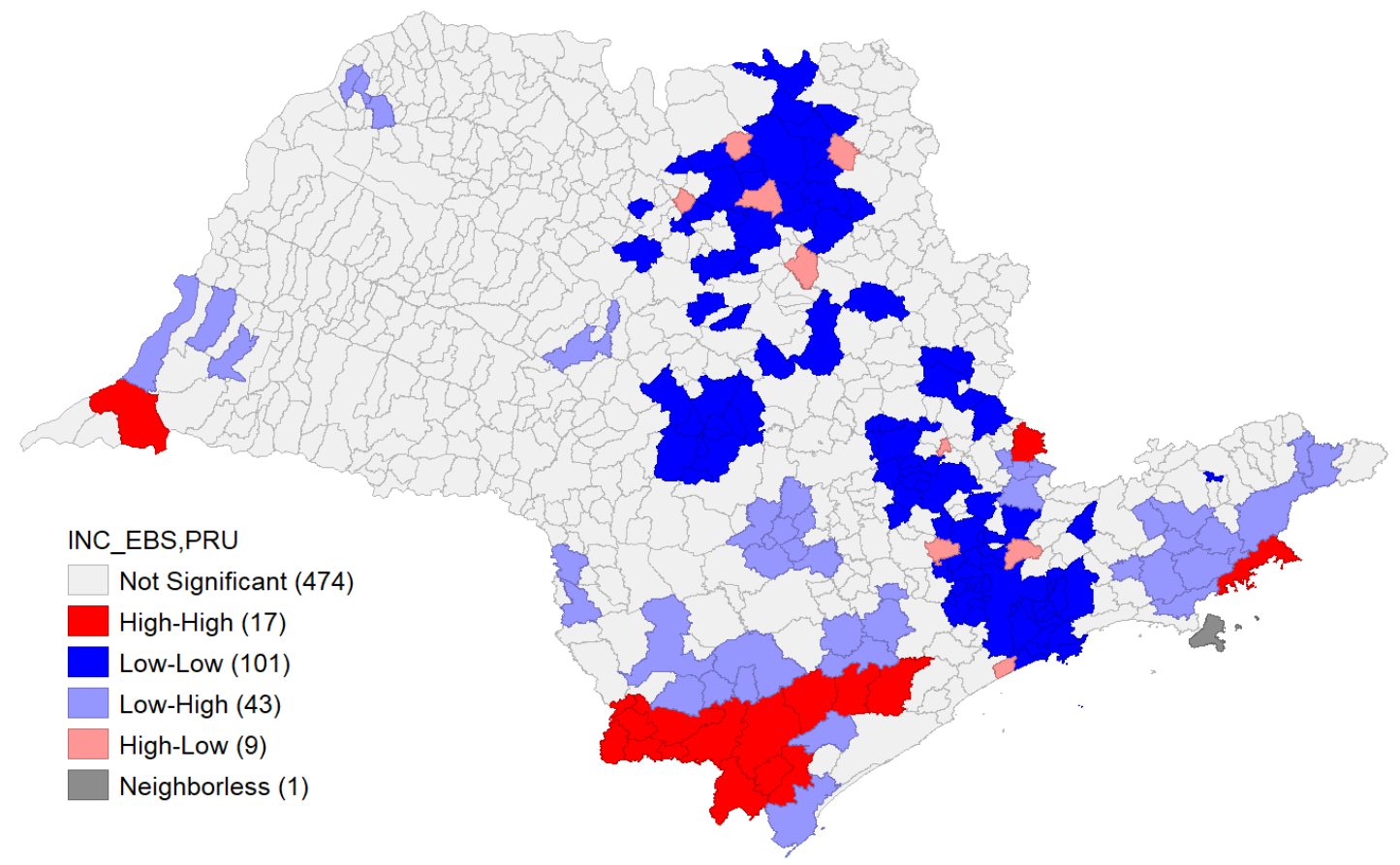

Fonte: Elaborado pelos autores (2021).

O aglomerado formado por municípios com alta taxa de incidência de LTA, espacialmente associados a municípios com alta taxa de população rural $(\mathrm{H}-\mathrm{H})$ encontra-se fortemente concentrado no Vale do Ribeira, geograficamente coincidente aos aglomerados $\mathrm{H}-\mathrm{H}$ (associação espacial positiva entre LTA e PCV, Figura 8) e HL (associação espacial negativa entre LTA e RPC, Figura 7). Além deste aglomerado $\mathrm{H}-\mathrm{H}$, com 14 municípios contíguos no Vale do Ribeira, identificamos também, outros três municípios isolados no aglomerado H-H do mapa da Figura 9: Teodoro Sampaio, situado no Pontal do Paranapanema; Socorro, na Mantiqueira Ocidental; e Ubatuba, localizado no litoral norte de São Paulo.

Constatamos ainda, um grande aglomerado com 101 municípios (L-L), que apresentou associação espacial entre baixa taxa de incidência de LTA e baixo percentual de população rural. Este aglomerado é representado por municípios localizados nas regiões metropolitanas de São Paulo, Campinas e Ribeirão Preto, e mesorregiões de Bauru e Piracicaba. Observa-se ainda no mapa da Figura 9, o aglomerado H-L, caracterizado pela associação espacial entre alta taxa de incidência 
de LTA e baixo percentual de população rural. Este aglomerado é formado pelos municípios de Jaborandi, Nuporanga, Pitangueiras, Guatapará e Paraíso, localizados no norte do estado, e Cabreúva, Mairiporã, no Planalto Atlântico, e Mongaguá no litoral sul do estado.

\section{DISCUSSÃO}

Na série histórica de casos de LTA utilizada neste artigo, observamos que o ano de 2015 apresentou o maior desvio positivo em relação à mediana, resultado do grande número de casos notificados neste ano. Um dos fatores que poderia explicar este aumento de casos seria o fenômeno de escassez de chuvas observado nos verões 2013-2014 e 2014-2015, responsável pela escassez hídrica que ocorreu no estado de São Paulo em 2015. Nestes verões, foram observadas anomalias pluviométricas no Sudeste do país, com totais de chuva abaixo da média climatológica, observados entre outubro de 2014 e janeiro de 2015 (MARENGO et al. 2015).

Em estudo realizado no município de Pedro de Toledo, no Vale do Ribeira, Domingos et al. (1998) observaram que, após períodos de precipitação pluviométrica intensa, a população de Leishmania intermedia tende a reduzir drasticamente. Isto se deve, segundo os autores, ao fato de os criadouros da espécie estarem associados ao solo situado sob o dossel compacto da mata. Esta queda nas precipitações no verão 2014-2015 pode ter contribuído para o aumento na reprodução dos mosquitos flebotomíneos, vetores da LTA.

O aglomerado de alto risco de LTA situado no Vale do Ribeira distribui-se por duas províncias geomorfológicas: a Província Costeira e a Província do Planalto Atlântico (IPT, 1981). Neste aglomerado, a Província Costeira subdivide-se em duas zonas: Serrania Costeira, formada pelas subzonas Serra de Paranapiacaba e Serrania do Ribeira; e, a zona da Morraria Costeira. A Província do Planalto Atlântico subdividese em duas zonas: Planalto de Guapiara e Planalto do Alto Turvo. A região do vale do Ribeira é uma das maiores áreas de remanescentes de mata atlântica do Brasil, cujas características históricas de uso são marcadas por atividades rurais e a presença de comunidades tradicionais (CARLOS; IGNATTI; ROSA, 2015).

A pluviosidade média anual deste aglomerado varia entre $1.400 \mathrm{~mm}$ e 3.500 $\mathrm{mm}$; as temperaturas médias anuais oscilam entre $15^{\circ} \mathrm{C}$ e $22^{\circ} \mathrm{C}$, e a média da umidade relativa do ar situa-se acima de $80 \%$ (CIIAGRO, 1974). A cobertura vegetal primitiva é caracterizada pela fitofisionomia da Floresta Ombrófila Densa na Serra de Paranapiacaba e Serrania do Ribeira; Floresta Ombrófila Mista no Planalto de Guapiara, e Floresta Ombrófila Densa de Terras Baixas na Morraria Costeira (SÃO PAULO, 2020). Os municípios deste aglomerado apresentam percentuais de cobertura vegetal nativa entre $25 \%$ e $94 \%$, proporção de população rural entre $23 \%$ e $74 \%$ e renda per capita inferior a $\mathrm{R} \$ 480,00$ (SEADE, 2020).

Mayo et al. (1998) estudaram uma área de transmissão da LTA no Planalto Atlântico, na zona rural do município de Itapeva, e identificaram a presença de 14 espécies de flebotomíneos. Segundo os autores, a existência desta alta variedade de vetores da doença na área se deve, provavelmente, à diversificação das formas de relevo e da vegetação. Além disto, a pesquisa constatou a ocorrência das mesmas espécies, tanto no ambiente familiar como na floresta, em função da proximidade entre os mesmos.

A associação entre a LTA e atividades antrópicas desenvolvidas em áreas com alto grau de vegetação, e ambientes periurbanos, são locais com alto fator de risco da 
doença (GUERRA et al., 2015; MELO; ROSSONI; TEODORO, 2018). Além da cobertura vegetal, fatores climáticos como temperatura e precipitação também podem estar associados à sua incidência (VALDERRAMA-ARDILA et al., 2010; MOLALLO et al., 2015).

Esta afirmação talvez possa explicar a existência de outro aglomerado de alto risco do Extremo Oeste do estado, identificado em nosso estudo no Planalto Ocidental, onde a precipitação média anual é inferior à do aglomerado do Vale do Ribeira.

O aglomerado de alto risco do Extremo Oeste ocupa as áreas de altitudes mais baixas da província geomorfológica do Planalto Ocidental. A pluviosidade média anual varia entre $1.100 \mathrm{~mm}$ a $1.200 \mathrm{~mm}$ anuais, com temperaturas médias superiores a $22^{\circ} \mathrm{C}$ e umidade relativa do ar média oscilando entre $75 \%$ a $80 \%$. Neste aglomerado de alto risco, a fitofisionomia restringe-se aos fragmentos de Floresta Estacional Semidecidual. Nos municípios deste aglomerado, os percentuais de cobertura vegetal primitiva são inferiores aos observados no aglomerado do Vale do Ribeira, variando entre $9 \%$ a $25 \%$. A proporção de população rural variou de $14 \%$ a $74 \%$, e a renda per capita é superior a $R \$ 550,00$.

Fonseca et al. (2014) analisaram o perfil epidemiológico da LTA no município de Teodoro Sampaio, localizado no pontal do Paranapanema, e constataram a ocorrência de transmissão periurbana. Os autores concluíram que os principais focos de vetores se localizavam no distrito do Parque Estadual do Morro do Diabo, reserva do bioma de mata atlântica, onde ocorre fitofisionomia da Floresta Estacional Semidecidual.

Em nosso trabalho, constatamos a existência de associação espacial positiva entre a taxa de incidência de LTA e o percentual de cobertura vegetal nativa no município. Este achado pode ser explicado pela maior possibilidade de a população residir próximo a áreas florestadas, e, desta forma, ter contato com insetos vetores da doença. Barretto et al. (1981) já haviam constatado a transmissão da LTA em trabalhadores de fazendas na Bahia, onde ocorria a derrubada da floresta para o plantio do cacau, impulsionada por incentivos governamentais.

Pesquisa realizada por Lonardoni et al., (2006) no norte do estado do Paraná, identificou um elevado número de flebotomíneos em domicílio e peridomicílio, em um assentamento rural no município de Mariluz. Os autores associaram este fato à derrubada de árvores para construção de moradias próximas à fragmentos de matas. Além disto, a expansão das áreas plantadas com soja, milho, cana de açúcar e pastagens, próximas às matas, tem contribuído também para o aumento no número de casos de LTA no estado.

Em nosso estudo, identificamos a associação espacial positiva e significante entre a taxa de incidência da LTA e a proporção de população rural no município. Nossos resultados são corroborados por Silva; Latorre; Galati (2010), que constataram, no Vale do Ribeira, associação entre a taxa de incidência da doença, o grau de urbanização e a renda per capita, e afirmaram que estes fatores favorecem a exposição da população aos vetores da LTA. Temponi et al. (2018) identificaram forte associação entre casos de LTA, população rural e deficiência nas condições de saneamento básico em circuitos espaciais de produção agrícola em Minas Gerais.

Santos et al. (2000) realizaram uma investigação socioeconômica na população residente em fazendas na localidade de Corte de Pedra, município de Tancredo Neves, Bahia, uma área considerada endêmica para a LTA, e onde foi constatada a transmissão intradomiciliar e peridomiciliar. Os resultados mostraram 
que $66,7 \%$ das famílias recebiam um salário mínimo ou menos, $44,1 \%$ eram lavradores e $57,8 \%$ dos domicílios eram habitados por 5 ou mais pessoas.

\section{CONCLUSÕES}

O uso de métodos e técnicas de análise geoespacial baseadas no paradigma de autocorrelação espacial, permitiu identificar e mapear aglomerados de alto e baixo risco de leishmaniose tegumentar americana no estado de São Paulo.

O principal aglomerado de alto risco de LTA localiza-se no Vale do Ribeira, cuja estrutura da paisagem caracteriza-se pela associação entre a fitofisionomia da Floresta Ombrófila Densa, e terrenos da Serra de Paranapiacaba e Serrania do Ribeira; fitofisionomia da Floresta Ombrófila Mista em terrenos do Planalto de Guapiara; e Floresta Ombrófila Densa de Terras Baixas em terrenos da Morraria Costeira.

O ano de 2015 apresentou o maior desvio positivo no número de casos, provavelmente em razão da anomalia pluviométrica abaixo da média histórica, verificada no verão 2014-2015, o que teria propiciado a melhor reprodução dos flebotomíneos, pela preservação de criadouros.

As taxas mais altas de incidência estão associadas espacialmente a municípios com alto percentual de população rural, alto percentual de cobertura vegetal primitiva e baixa renda per capita. Portanto, está claro que a leishmaniose tegumentar americana no estado de São Paulo é um problema socioambiental grave e delicado, que afeta, em sua maioria, populações mais pobres que trabalham e residem em domicílios localizados junto às florestas, e que, possivelmente, podem apresentar dificuldades de acesso aos serviços básicos de saúde.

\section{REFERÊNCIAS BIBLIOGRÁFICAS}

ALI-AKBARPOUR, M.; MOHAMMADBEIGI, A.; TABATABAEE, S. H.; HATAM, G. Spatial analysis of eco-environmental risk factors of cutaneous leishmaniasis in Southern Iran. Journal of Cutaneous and Aesthetic Surgery, v. 5, n. 5, p.30-35, 2012.

ANSELIN, L. Quantile local spatial autocorrelation. Letters in Spatial and Resource Sciences. V. 12, p. $155-166,2019$.

ANSELIN L., SYABRI I., KHO Y. GeoDa: An Introduction to Spatial Data Analysis. In: FISCHER M., GETIS A. (eds) Handbook of Applied Spatial Analysis. Berlin: Springer, 2010. p. 73-89.

BARRETTO, A. C.; CUBA, C. A. C.; MARSDEN, P. D.; VEXANAT, J. A.; BELDER, M. Características epidemiológicas da leishmaniose tegumentar americana em uma região endêmica do estado da Bahia, Brasil. Bol. Of Sanit Panam, v. 90, n. 5, p. 415424, 1981.

BEZERRA, J. M. T.; ARAÚJO, V. E. M.; BARBOSA, D. S.; MARTINS-MELO, F. R.; WERNECK, G. L.; CARNEIRO, M. Burden of leishmaniasis in Brazil and federated units, 1990-2016: Findings from Global Burden of Disease Study 2016. PLoS Negl Trop Dis, v. 12, n.9, p. 1-19, 2018.

BRASIL, Ministério da saúde. 2013. Manual de Vigilância da Leishmaniose Tegumentar Americana. Ministério da Saúde. 2. ed. Brasília: Secretaria de Vigilância em Saúde, 2013, 180 p. 
BRASIL, Ministério da Saúde. Secretaria de Vigilância em Saúde. Departamento de Vigilância Epidemiológica. 2006. Atlas de leishmaniose tegumentar americana: diagnósticos clínico e diferencial. Brasília: Editora do Ministério da Saúde, 2006, $136 \mathrm{p}$.

CARLOS, M. M.; IGNATTI, C.; ROSA, M. R. Leishmaniose tegumentar americana: incidência no Vale do Ribeira/São Paulo, Brasil. Revista Recien, v. 5, n. 15, p. 3-10, 2015.

CENTRO INTEGRADO DE INFORMAÇÕES AGROMETEOROLÓGICAS (CIIAGRO). Mapa de umidade relativa anual, São Paulo, CIIAGRO, 1974.

CENTRO INTEGRADO DE INFORMAÇÕES AGROMETEOROLÓGICAS (CIIAGRO). Mapa de isotermas anuais, São Paulo, CIIAGRO, 1974.

DOMINGOS, M. F.; CARRERI-BRUNO, G. C.; CIARAVOLO, R. M. C.; GALATI, A. B.; WANDERLEY, D. M. V.; CORRÊA, F. M. A. Leishmaniose tegumentar americana: flebotomíneos de área de transmissão, no município de Pedro de Toledo, região sul do Estado de São Paulo, Brasil. Revista da Sociedade Brasileira de Medicina Tropical, v. 31, n. 5, p. 425-432, 1998.

FONSECA, E. S.; ANDREA, L. A. Z.; TANIGUCHI, H.H.; HIRAMOTO, R. M.; TOLEZANO, J. E.; GUIMARÃES, R. B. Spatial epidemiology of American cutaneous leishmaniasis in a municipality of west São Paulo State, Brazil. J Vector Borne Dis, v.51, p.271-275, 2014.

GOSCH, C. S.; MARQUES, C. P.; RESENDE, B. S.; SOUZA, J. S.; ROCHA, R. A. S.; LOPES, D. S. S.; GOSCH, M. S.; DIAS, F. R.; DORTA, M. L. American tegumentary leishmaniasis: epidemiological and molecular characterization of prevalent Leishmania species in the State of Tocantins, Brazil, 2011-2015. Rev Inst Med Trop São Paulo, v.59, p. 1-11, 2017.

GUERRA, J. A. O.; MACIEL, M. G.; GUERRA, M. V.F.; TALHARI, A. C.; PRESTES, S. R.; FERNANDES, M. A.; DA-CRUZ, A. M.; MARTINS, A.; COELHO, L. I. A.R.; ROMERO, G. A.S.; BARBOSA, M. G. V. Tegumentary leishmaniasis in the State of Amazonas: what have we learned and what do we need?. Rev Soc Bras Med Trop, v. 8, p. 12-19, 2015.

INSTITUTO BRASILEIRO DE GEOGRAFIA E ESTATÍSTICA (IBGE). 2020. Disponível em: www.ibge.gov.br/geociencias/downloads-geociencias.

INSTITUTO DE PESQUISAS TECNOLÓGICAS DO ESTADO DE SÃO PAULO (IPT). Mapa geomorfológico do Estado de São Paulo, São Paulo, IPT, 1981.

LAINSON R. Epidemiologia e ecologia de leishmaniose tegumentar na Amazônia. Hiléia Méd (Belém), v. 3, p. 35-40,1981.

LONARDONI, M. V. C.; SILVEIRA, T. G. V.; ALVES, W. A.; MAIA-ELKHOURY, A. N. S. MEMBRIVE, U. A.; MEMBRIVE, N. A; RODRIGUES, G.; REIS, N.; ZANZARINI, P. D.; ISHIKAWA, E.; TEODORO, U. Leishmaniose tegumentar americana humana e canina no Município de Mariluz, Estado do Paraná, Brasil. Cad. Saúde Pública, v. 22, n. 12, p.2713-2716, 2006.

MARCHI, M. N. A.; CALDART, E. T.; MARTINS, F. D. C.; FREIRE, R. L. Spatial analysis of leishmaniasis in Brazil: a systematized review. Rev Inst Med Trop São Paulo, v. 61, p. 1-7, 2019. 
MARENGO, J. A.; NOBRE, C. A.; SELUCHI, M. E.; CUARTAS, A.; ALVES, L.M.; MENDIONDO, E. M.; OBREGÓN, G.; SAMPAIO, G. A seca e a crise hídrica de 20142015 em São Paulo. Revista USP, n. 106, p. 31-44, 2015.

MARTINELLI, M. Clima do Estado de São Paulo. Confins [Online], v.8, 2010.

MAYO, R. C.; CASANOVA, C.; MASCARINI, L. M.; PIGNATTI, M. G.; RANGEL, O.; GALATI, E. A. B.; WANDERLEY, D. M. V.; CORRÊA, F. M. A. Flebotomíneos (Diptera, Psychodidae) de área de transmissão de leishmaniose tegumentar americana, no município de Itupeva, região sudeste do estado de São Paulo, Brasil. Revista da Sociedade Brasileira de Medicina Tropical, v. 31, n. 4, p. 339-345, 1998.

MELO, H. A.; ROSSONI, D. F.; TEODORO, U. Effect of vegetation on cutaneous leishmaniasis in Paraná, Brazil. Mem Inst Oswaldo Cruz, v, 113, n.6, p. 1-7, 2018.

MENDES, C. S.; COELHO, A. B.; FÉRES, J. G; SOUZA, E. C.; CUNHA, D. A. Impacto das mudanças climáticas sobre a leishmaniose no Brasil. Ciência \& Saúde Coletiva, v. 21, n. 1, p. 263-272, 2016.

MOLLALO, A.; ALIMOHAMMADI, A.; SHIRZADI, M. R.; MALEK, M. R. Geographic Information System-Based Analysis of the Spatial and Spatio-Temporal Distribution of Zoonotic Cutaneous Leishmaniasis in Golestan Province, North-East of Iran. Zoonoses and Public Health, v. 62, p. 18-28, 2015.

MORETTI, A. I. P.; FERREIRA, M. C. Dinâmica espacial e regionalização da Leishmaniose Tegumentar Americana no estado de São Paulo. Geografia (Rio Claro. Impresso), v. 36, n.3, p. 453-468.

NEGRÃO, G. N.; FERREIRA, M. E. M. C. Considerações sobre a leishmaniose tegumentar. Revista Percurso - NEMO, v. 6, n. 1, p. 147 - 168, 2014.

PAVLOSKIY, E. N. The theory on natural focality of transmissive diseases of man. Ruk. Parazitol. Cheloveka, v.2, p. 915-38, 1948.

PAVLOSKY, E. N. Natural Nidality of Transmissible Diseases with Special Reference to the Landscape Ecology of Zooanthroponselllinois: University of Illinois Press, 1966, 261p.

REISEN, W. K. Landscape epidemiology of vector-borne diseases. Annu Rev Entomol, v.55, p.461-483, 2010.

RESENDE, S. M. Análise eco-epidemiológica da leishmaniose tegumentar americana em uma área endêmica da microrregião de Caratinga, Minas Gerais (Brasil), submetida a ensaio comunitário com vacina anti LTA. 2004. 11 p. (Dissertação de Mestrado). Universidade Federal de Minas Gerais, Escola de Veterinária, 2004.

RODRÍGUEZ, E-M.; DÍAZ, F.; PERÉZ, M-V. Spatio-temporal clustering of American Cutaneous Leishmaniasis in a rural municipality of Venezuela. Epidemics, v. 5, p. 1119, 2013.

ROSS, J. L. S.; MOROZ, I. C. Mapa geomorfológico do estado de São Paulo. Revista de Departamento de Geografia, v. 10, p. 41-58, 1996.

SANTOS, J. B.; LAUAND, L.; SOUZA, G. S.; MACÊDO, V, O. Fatores socioeconômicos e atitudes em relação à prevenção domiciliar da leishmaniose tegumentar americana, em uma área endêmica do sul da Bahia, Brasil. Cad. Saúde Pública, v.16, n.3, p701-708, 2000. 
SÃO PAULO. Inventário florestal do estado de São Paulo: mapeamento da cobertura vegetal nativa. Instituto Florestal, São Paulo, 2020, 40 p.

SHIMABUKURO, P. H. F.; SILVA, T. R. R.; FONSECA, F. O. R.; BATON, L. A; GALATI, E. A. B. Geographical distribution of American cutaneous leishmaniasis and its phlebotomine vectors (Diptera: Psychodidae) in the state of São Paulo, Brazil. Parasites \& Vectors, v. 3, n. 121, p. 1-12, 2010.

SILVA, A. F.; LATORRE, M. R. D. O.; GALATI, E. P. B. Fatores relacionados à ocorrência de leishmaniose tegumentar no Vale do Ribeira. Revista da Sociedade Brasileira de Medicina Tropical, v. 43, n. 1, p. 46-51, 2010.

SISTEMA DE INFORMAÇÃO DE AGRAVOS DE NOTIFICAÇÕES (SINAN). 2020. Brasil, Ministério da Saúde. Disponível em: http://www2.datasus.gov.br/DATASUS/index.php?area=0203\&id=29878153.

SISTEMA ESTADUAL DE ANÁLISE DE DADOS (SEADE). 2020. Disponível em: www.seade.gov.br.

TEMPONI, A. O. D.; BRITO, M. G.; DINIZ, M. L. F.; FERRAZ, M. L.; DINIZ, S. A.; SILVA.; M. X.; CUNHA, T. N. Ocorrência de casos de leishmaniose tegumentar americana: uma análise multivariada dos circuitos espaciais de produção, Minas Gerais, Brasil, 2007 a 2011. Cadernos de Saúde Pública, v. 34, n. 2, 2018.

VALDERRAMA-ARDILA, C.; ALEXANDER, N.; FERRO, C.; CADENA, H.; MARÍN, D.; HOLFORD, T. R.; MUNSTERMANN, L. E.; OCAMPO, C. B. Environmental Risk Factors for the Incidence of American Cutaneous Leishmaniasis in a Sub-Andean Zone of Colombia (Chaparral, Tolima). Am. J. Trop. Med. Hyg, v. 82, n. 2, p.243-250, 2010.

WHO - World Health Organization. 2010. Control of the leishmaniases: report of a meeting of the WHO Expert Committee on the Control of Leishmaniases, Geneva, 2226 March 2010, 185p. 